\title{
Technology and peer review: the open and participatory dimension
}

\author{
Laura Fedeli
}

University of Macerata, Italy, laura.fedeli@unimc.it

\begin{abstract}
Peer review is a consolidated procedure in the academic context and its process affects various range of research outputs from project funding applications to manuscript publication. Peer review can be developed through modalities that imply a different level of transparency in the relationship between anonymity of the author and the reviewer/s.

With the development of social media and the growth of scientific online communities, new forms of peer review have acquired a recognised value, matching the need of the academy to rely on selected reviewers and the need of the prospective author to get a richer feedback from a variety of scholars through different means, open comments and/or discussion fora, and always accessible online.

Hybrid forms of review, which can integrate a formal peer review with an open comment opportunity on the Web, proved successful for both improving the author's draft and enhancing its chances of publication and for the reviewers who can use this valuable activity to enrich their reputation by collecting and showing their reviews as research output. In this framework, quality, transparency and reputation acquire new nuances in their connection with the process of research validation.
\end{abstract}

Keywords: peer evaluation; research, technology.

\section{Introduction}

The development of online environments and tools born in the framework of Web 2.0 that aimed at valorising and optimising the interaction dynamics amongst users, the sharing processes and the promotion of artefacts in a community scenario rationale (Fedeli, 2012) represents a privileged landscape for the so-called digital scholar. Externalisation and pervasivity are the dimensions of an augmented presence of the researcher in the development of his/her scientific activity.

By introducing the phrase 'digital scholar', Weller (2011) underlined that being a researcher in the digital age implies a change in the attitudes that reifies in embracing values and ideologies of the 'open access' 1 trend and in recognising the impact of peer collaborative and networking activities. In fact, the sound change is not just affected by the quantity of available resources in the Web and the efficacy of networking processes but is tied to the legitimacy they acquired in the academic context.

This contribution focuses on the concept of peer review and on the challenges and opportunities originating from the massive use of the Web and the different online publishing modalities. In fact, social media has created a range of alternatives to the classical process of peer review.

The validation of the research output is, thus, currently acquiring a new participatory dimension developed in academic online communities. As described in the contribution, this dimension can help overcoming some critical aspects of the classical process of peer review such as the overload for reviewers, the lack of motivation in embracing a review request and the subsequent delay in publishing.

There will be a description of some current options activated by scientific communities to achieve validation of the research outputs in their different phases of development. The participatory dimension of peer review can, in fact, affect the pre-publication and the post-publication step as well.

Quality and validation are concepts accompanied by a new self-regulation for the author, a reflection process that can be fostered by the open and public exchange with experts with whom it is possible to share an interactive review.

In such a way, the reviewer's status is enriched, acquiring new nuances from a 'decider' to a 'critical friend', a profile that is more directed in supporting the author and the prospective audience of the research output.

\footnotetext{
${ }^{1}$ The phrase 'open access' is related to both technical aspects (the use of open software and standards), and the trend of sharing content (articles, books, research raw data), a process that is simplified by the use of tools and environments of Web 2.0 in which collaboration is a common principle.
} 


\section{Peer review: a brief overview}

The origin of peer review can be traced back to the English academic societies of the eighteenth century, even if in the context of journal publication we have the first quality assurance system in the form of peer review already in the seventeenth century with the Journal des Sçavans and the Philosophical Transactions of the Royal Society, published in 1665 in France and England, respectively (The British Academy, 2007).

But peer review became a consolidated procedure in the academic context only in the second middle of the twentieth century (Research Information Network, 2010).

Peer review validation is at the basis of the funding application procedures for research projects and a necessary condition for the publication of research output that satisfies quality standards. There exists different procedures and criteria to satisfy in the peer review processes, managed by institutions that analyse and fund research proposal applications and journals that publish the research output.

The peer review has been the object of analysis by several governmental institutions. In 1989, the 'Boden Report' was published in the United Kingdom in reply to the criticalities highlighted by the Secretary of State for Education who had underlined the disadvantages connected to the peer review as a too bureaucratic and time-consuming process. The document represents the result of the work developed by a group elected by the Board for the Research Councils and highlights how the peer review is the only option to assess research. Even if an improvement of the process was recommended, no clues were offered to identify what means and methodologies should have been used to reach that goal. The insistence on the value of peer review was reinforced by the report of the Royal Society (1995) and in the more recent 'Report of the Research Councils UK Efficiency and Effectiveness of Peer Review Project' (Research Councils UK, 2006). It is interesting that in publishing 'Peer Review - An Assessment of Recent Developments', the Royal Society is recognising the ductility of the peer review that is meant as a non-static process.

Even if the literature regarding peer review effects and criticalities is rich (Jefferson et al., 2002), the roles assigned to these procedures have the historically accredited aim of reaching the following advantages connected to the quality of the scientific output:

- to offer the author the chance of improving his work: from an investigation run by Ware and Monkman (2008), $90 \%$ of the sample (3040 academic professionals) stated that the peer review was an opportunity to correct both typographical errors and conceptual and formal aspects;

- too act as a filter for the community: peer review can, for example, help place an article in the proper journal by working as a selection tool or filter; in this way, the reader can rely on the different journal's distinction;

- to reduce the spread of illegal conduct (plagiarism, fraud): this aspect is being widely discussed by the scientific community to identify the specific responsibilities of the reviewers and the editors in the detection of author's misconduct.

Three main modalities of peer review can be identified according to the transparency concept (relationship between anonymity of the author and of the reviewer):

- $\quad$ single-blind review: the identity of the author is clear to the reviewer who keeps, however, their anonymity from the author. Such options have some disadvantages (Budden et al., 2008; Peters \& Ceci, 1982; Wennerås \& Wold, 1997) mainly in relation to the risk that the reviewer can be influenced by prejudices (gender, culture/language, role, etc.);

- double-blind review: the identity of both the author and the reviewer is hidden. This procedure would reduce the risk of detrimental and unethical behaviours by the reviewer even if, in many cases, it is clearly difficult to successfully anonymise a proposal (Lane, 2008);

- Open review: the identity of both the author and of the reviewer is transparent to each other. The desirable benefits of this solution often meet not only the barriers of the editors but also the diffidence of authors and reviewers (Research Information Network, 2015; Ware \& Monkman, 2008);

An investigation run by the 'Publishing Research Consortium' in the United Kingdom, representing associations and editors interested in the study of the communication processes in academic research, highlights the perceptions of a selected sample (3,040 participants amongst authors, reviewers and editors) regarding the different modalities of peer review (Ware \& Monkman, 2008). Two macro areas are the focus of the study: the opinions expressed by the sample and the practices connected to peer review.

A high percentage of participants (more than $80 \%$ ) perceived peer review as a tool of control that helps avoid situations of anarchy in the publishing context, and they also valorise its function of validating the quality of the research and facilitating the author to improve his/her own output. More than $50 \%$ of the sample expressed the need to optimise the process of revision, showing a clear preference for the 'double-blind' procedure over the 'single-blind' and 'open' review.

\section{The Web and the peer review process: new hybrid options}

In the three cases mentioned earlier, we can refer to 'classical' peer review, that is, the process that develops before the publication because it is the conditio sine qua non of the approval. Is this process reliable? Does the presence of a 
peer review before publication represent a quality assurance step? The discussion around this aspect is currently open in the scientific community. A different position is the one that promotes the 'self-appointed post hoc peer review' and that believes that the absence of the mediation of an editor (who selects reviewers and assesses their reviews) does not affect the validity of a review process run after the publication by a self-appointed expert.

'Post-publication' peer review may actually refer to a set of different procedures (Research Information Network, 2015) from the review that takes place after a first publication (pre-print), but not submitted to a formal review of the comments published on the editors' websites or social media channels after the review. Comments can be either moderated by editors and curators or published on the Web without any mediation. In the first case, post-publication represents an example of such a trend to move from a 'filter-then-publish' model to a 'publish-then-filter' one.

In the present paragraph, a set of options that relies on a different concept of shared responsibility in peer review will be described. It is not just a matter of private answerability of the author to the reviewer or answerability of the reviewer to the editor. Anonymity, responsibility and reputation are strictly connected in the peer review process, and it is undeniable that any attempt of innovation in the system opens up complex implications.

With the development of the Web, the peer review process was the object of various experimentations both in terms of spaces (editorial committee versus online communities) and time (extended time versus real time) and in terms of modalities (blind versus open review) and phases of development (pre- and post-publication).

The precursor of the concept of 'open access' and 'pre-publication review' is the electronic archive 'arXiv' (http://arxiv.org), founded in 1999 by Paul Ginsparg at the laboratories of Los Alamos in New Mexico and currently managed by the Cornell University Library. ArXiv was developed to gather scientific articles that can be commented on by the members of the community before their publication in a journal.

Environments such as arXiv satisfy, in a collective and participatory way, the need for exchange amongst scholars. Before the advent of the Internet, an article was sent to a few reliable colleagues to collect useful ideas and suggestions before submitting the work to a journal editor to undergo a peer review, now that restricted circle of friends does not have any definite border. The chance to receive comments in a pre-publication phase does not replace the peer review but enriches it. A similar recent example is the platform 'ResearchGate' (https://www.researchgate.net) developed in 2008 with the objective of making researchers share not only their publications but also their work in progress and raw data in order to receive feedback from the community before publication. The option of 'open review' was introduced in 2014 and it affects both the pre- and post-publication phases.

The pre-publication concept was also adopted by some educational journals. The British Journal of Educational Technology (BJET) offers the author the opportunity to ask for support during the production of the article. If requested, in fact, the editorial committee can match the so-called 'critical friend' to the author to help him/her during the writing process and, in case, also offer support when the article has already been submitted to a peer review and needs to be revised by the author. The practice activated by the BJET is surely innovative and creates a new profile, a mediator between the author and the reviewer who does not replace the roles of the reviewer but facilitates the preparation process and the revision, if needed, according to the feedback of the review.

There are cases in which the integration between a formal review and a pre- and post-publication comment system is systematic and suggested by the editorial guidelines of the journal. These hybrid forms of peer review aim to open the dialogue between the author and the scientific community (open review) whilst keeping the classical form of validation (blind review) by selected experts assigned by the journal.

An exemplar case is the International Journal for Innovation and Quality and in Learning (INNOQUAL) formed in 2012 within the EFQUEL framework (European Foundation for Quality in E-Learning). The review process implies a first step of open review run online by interested readers and that is organised around online discussions that can extend their action via social media and, in some cases, through a face-to-face event and a second step of classical double-blind review developed by experts.

As declared by the journal editorial committee, this hybrid system was inspired by the Atmospheric Chemistry and Physics (ACP) journal that applies a 'multi-stage open peer review' (Pöschl, 2012).

Actually, a pioneering experience of a multi-stage open review is to be found back in 1996 with the Journal of Interactive Media in Education (JIME), an e-journal targeted at researchers and practitioners in the field of educational technology. The attempt to overcome the boundaries of classical peer review through the use of technologies was, in this case, connected to the opportunity to create a computer-supported collaborative argumentation environment to 'rethink and redesign the process of scholarly debate at the heart of journal reviewing' (Sumner \& Shum, 1996).

The review model activated by JIME mixed pre-publication and post-publication review with a first private open peer review through a dedicated website to make authors and reviewers (and editors if needed) interact and, at a later stage, the publication of the article for a public broad debate opportunity. After the author's review, the editor verifies the article and edits the review debate in order to publish both and keep them open for further commentary (Shum \& Sumner, 2001).

The multi-stage review process is no longer present in the journal editorial policies where just a double-blind review process is mentioned. According to Pöschl (2012), the main downside of the JIME case is to be identified in the lack of integration between the pre- and post-publication, because the articles are made available to the public audience just after a private open peer review step and a first round of approval by the editor.

In ACP, instead, the different phases of validation start with a pre-screening (developed by the editor) and the subsequent publication as a 'discussion paper' in a forum for a period of eight weeks. During this time, reviewers 
selected by the editorial committee and other members of the scientific community can comment and provide their feedback. This opportunity of exchange is followed by a classical process of peer review. In consideration of the feedback received, the author can decide to continue with the review or to revise the work and submit it later. The whole procedure implies that the discussions are archived and always available and citable; this aspect is relevant to recognising the value of the review as a scientific output and increasing the quality of the system of validation. The publication of the discussion fosters a sort of self-regulation for the author who can acquire the motivation to further develop his/her work, improving it before the final submission. The success of such a system is not only in the integrated structure but also in the choice of having the classical peer review after the discussion step. A postpublication discussion can, in fact, have a minor impact on the motivation of both authors and audience. The scientific community may find it not as attractive as a pre-publication review. This architecture shows different aspects of reflection on the concepts of quality and transparency and, as underlined by Pöschl, on the fact that both are connected to the concept of self-regulation for the author. The researcher who wishes to submit a work to ACP is fostered to have a major attention and awareness, because the feedback collected in the first phase will be a relevant input to make corrections and decide whether or not to submit the definitive version.

A different solution is the so-called crowdsourcing peer review, a concept formed, thanks to an article published by Howe in the journal 'Wired' (2006), and coined using the existing concept of 'outsourcing', that is, the strategy through which firms do not perform some tasks (roles, services, productions) but assign them to external subjects in order to reduce costs. Crowdsourcing follows the same principle but relies on the 'wisdom of the crowd', that is, on the power of the Web communities and on the beliefs that you can get more from the involvement of a multiplicity of actors. But what is the connection with the scientific research and the academic community? If we analyse a simple definition of crowdsourcing as the one offered by Brabham (2012), as a 'model of production' based on problem solving and developed online where the collective intelligence of a community is required to deal with a specific task, this has a clear connection with strategies currently used by some researchers who look for the support of communities through the so-called 'call for participation' in order to receive precious input and validate his/her own work.

Even if such an approach was formed in different contexts (Buettner, 2015), its development has opened up debates on its efficacy and ability to replace or just accompany the classic peer review.

A primitive form of crowdsourcing is represented by the practices of blogging, activated by some authors who post in their blogs a draft of their research and create a 'conversational scholarship' (Lupton, 2014, 5). The blog or personal website become meta-reflection environments where you can get informal feedback from a vast and diverse audience.

\section{Conclusions}

In 1996, Sumner and Shum, describing the implementation of JIME, wrote 'What remains completely unsupported is the intellectual "meat" of scholarly publication, the review process itself. This remains a private affair involving a small number of reviewers, and in most paper-based journals, the gap between initial submission and article publication can be dragged out over a year. The potential of internet technologies for opening up and revitalising the scholarly debate process has yet to be realized'.

The growth of online scientific communities in Web 2.0 environments has reified the pioneering vision of JIME and fostered the development of additional/alternative forms of peer review that highlight new connections amongst the open discussion opportunities for authors and reviewers, the concept of quality and the validation of research output.

Following the experience of JIME, BJET and INNOQUAL, amongst the educational journals, and the case of ACP in the hard sciences, some aspects appear to be of of paramount importance for the success of the new hybrid forms of peer review activated, thanks to the collaborative features of the social Web: the architecture of the review process (integration of pre-publication and post-publication open review with classical blind review); the enhancement of the potential review feedback because of the exploitation of the 'wisdom of the crowd'.

The initiatives described here aim at involving a major number of actors with different profiles in the peer review process with the objective of overcoming some of the criticalities of the classical process of peer review managed by selected experts and editorial committees. In this new framework, the roles of reviewers are changing acquiring different nuances, and simultaneously, the value attributed to the review is enhanced. What happens if the review itself could become a research output? This is the idea launched by the start-up created by Publons in 2013 with the aim of optimising the work of the reviewer by making it a real research output. Each review done can be shown in the user profile can be 'measured' in terms of value and so be a means of reputation acquisition for the author of the review. In this way, the activity of reviewing can become a more rewarding practice from a professional perspective.

In the Publons vision, every review can have a 'digital object identifier' (DOI) and can be, in this way, easily identified and cited exactly as any research output (article, chapter, etc.):

'For academics, the assignment of DOIs helps to augment their publication record with a portfolio of reviews. This record can be used to strengthen applications for funding and jobs, and to collaborate in the academic community on a deeper level' (Publons, 2013).

The spread of hybrid forms of peer review show a multi-faceted need for the academy in keeping control of the review process and guaranteeing the quality of the final research output. But the innovative trend is the recognition of a 
more complex concept of quality that goes beyond the respect of standards through a formal selection of reviewers by editorial boards or a scientific committee and embrace, on one hand, the need of the author to be supported by the potential feedback of the 'crowd' and, on the other hand, the need of the reviewer to get recognition in terms of professional reputation for his/her activity.

\section{References}

Brabham, D. C. (2012). The myth of amateur crowds: A critical discourse analysis of crowdsourcing coverage. Information, Communication \& Society, 15(3), 394-410.

Budden, A. E., Tregenza, T., Aarssen, L. W., Koricheva, J., \& Leimu, R. (2008). Double-blind review favours increased representation of female authors. Trends in Ecology \& Evolution, 23, 4-6.

Buettner, R. (2015). A systematic literature review of crowdsourcing research from a human resource management perspective. Proceedings of the 48th Hawaii International Conference on System Sciences - (HICSS-48), (46094618). Kauai, Hawaii.

Fedeli L. (2012). Social media e didattica. Opportunità, criticità e prospettive. Lecce: Pensa Multimedia.

Howe, J. (2006). The rise of crowdsourcing. Wired, 14(6). Retrieved from http://goo.gl/vO7E7B.

Jefferson, T., Alderson, P. Wager, E., \& Davidoff, F. (2002). Effects of editorial peer review: A systematic review. Journal of the American Medical Association, 287, 2784-2786.

Lane, D. (2008). Double-blind review: Easy to guess in specialist fields. Nature, 452, 28.

Lupton, D. (2014). "Feeling Better Connected": Academics' Use of Social Media University of Canberra. Retrieved from http://goo.gl/OFBFaE.

Peters, D., \& Ceci, S. (1982). Peer-review practices of psychological journals: The fate of submitted articles, submitted again. Behavioral and Brain Sciences, 5, 187-255.

Pöschl, U. (2012). Multi-stage open peer review: scientific evaluation integrating the strengths of traditional peer review with the virtues of transparency and self-regulation. Frontiers in Computational Neuroscience, 6(July), 33. http://dx.doi.org/10.3389/fncom.2012.00033.

Publons, (2013). Publons Launches DOI Support For Peer Reviews. Retrieved from https://goo.gl/b9iElb.

Research Councils UK (2006). Report of the Research Councils UK Efficiency and Effectiveness of Peer Review Project. Retrieved from http://goo.gl/dlRb1n.

Research Information Network. (2010). Peer review. A guide for researchers. Retrieved from www.rin.ac.uk/peerreview-guide.

Research Information Network (2015). Scholarly Communication and Peer Review. The Current Landscape and Future Trends. Research Information Network. Retrieved from http://goo.gl/YMhzKb.

Shum, S. B., \& Sumner, T. (2001). JIME: An interactive journal for interactive media. First Monday, 6 (2). Retrieved from http://firstmonday.org/ojs/index.php/fm/article/view/836/745.

Sumner, T., Shum S. B. (1996). Open Peer Review \& Argumentation: Loosening the Paper Chains on Journals. Ariadne. Retrieved from http://www.ariadne.ac.uk/issue5/jime.

The British Academy. (2007). Executive Summary and Recommendations. Retrieved from http://goo.gl/SKkDEz.

The Royal Society. (1995). Peer Review - An assessment of recent developments. Retrieved from http://goo.gl/RH27TU.

Ware, M., \& Monkman, M. (2008). Peer review in scholarly journals: Perspective of the scholarly community - an international study. Retrieved from http://goo.gl/ThJCXp. 
Technology and peer review: the open and participatory dimension

Fedeli

Weller, M. (2011). The Digital Scholar. How technology is transforming scholarly practice. London: Bloomsbury Academic.

Wennerås, C., \& Wold, A. (1997). Sexism and nepotism in peer-review. Nature, 387, 341-3. 